\title{
Comparison of tocilizumab as monotherapy or with add-on disease-modifying antirheumatic drugs in patients with rheumatoid arthritis and inadequate responses to previous treatments: an open-label study close to clinical practice
}

\author{
Vivian P. Bykerk • Andrew J. K. Östör • José Alvaro-Gracia • Karel Pavelka • \\ José Andrés Román Ivorra • Winfried Graninger • William Bensen • \\ Michael T. Nurmohamed • Andreas Krause • Corrado Bernasconi • Maher Aassi • \\ Jean Sibilia
}

Received: 13 February 2014 /Revised: 18 December 2014 / Accepted: 22 December 2014 /Published online: 22 January 2015

(C) The Author(s) 2015. This article is published with open access at Springerlink.com

\begin{abstract}
This was an exploratory analysis comparing the safety and efficacy of tocilizumab monotherapy with those of tocilizumab in combination with disease-modifying antirheumatic drugs (DMARDs). Data were from a single-arm, nonrandomized, open-label, 24-week study in patients with rheumatoid arthritis in which patients with inadequate responses to DMARDs or tumor necrosis factor- $\alpha$ inhibitors received tocilizumab $8 \mathrm{mg} / \mathrm{kg}$ intravenously every 4 weeks plus methotrexate/other DMARD(s) combination therapy. If they were intolerant of methotrexate/other DMARD, patients received tocilizumab monotherapy. Effectiveness endpoints
\end{abstract}

Electronic supplementary material The online version of this article (doi:10.1007/s10067-014-2857-y) contains supplementary material, which is available to authorized users.

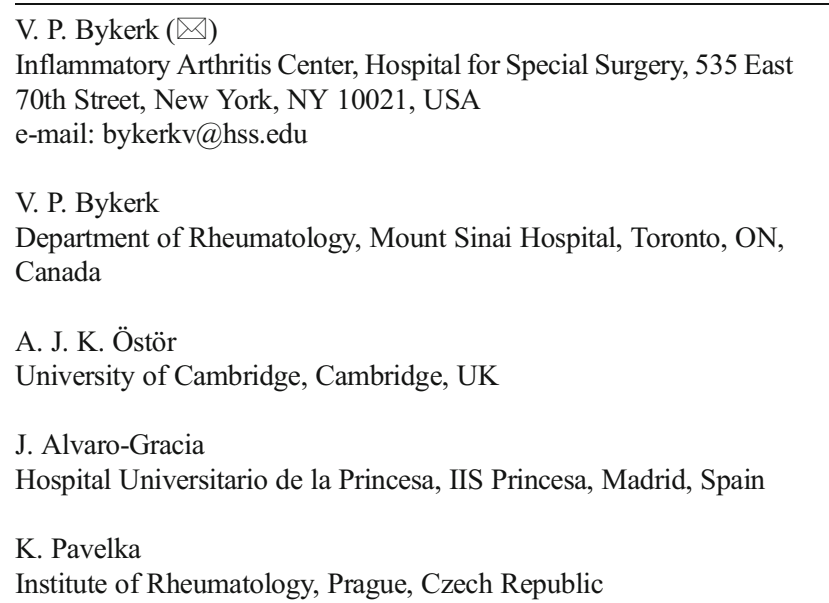

J. A. R. Ivorra

Hospital Universitario La Fe, Valencia, Spain included American College of Rheumatology (ACR) responses (ACR20/50/70/90) and disease activity score using 28 joints (DAS28). Of 1,681 patients, 239 received tocilizumab monotherapy, and 1,442 received combination therapy. Methotrexate was the most common DMARD (79 \%) used in combination therapy. The frequency of adverse events (AEs), serious AEs, and AEs leading to withdrawal were similar between tocilizumab monotherapy $(82.4,7.9$, and $5.4 \%$, respectively) and combination therapy $(76.6,7.8$, and $5.1 \%$, respectively). No differences in ACR20/50/70/90 responses were observed between treatment groups (66.9\%/

W. Graninger

Medical University of Graz, Graz, Austria

W. Bensen

St Joseph's Hospital/McMaster University, Hamilton, ON, Canada

M. T. Nurmohamed

VU University Medical Center, Amsterdam, Netherlands

A. Krause

Immanuel Hospital, Berlin, Germany

C. Bernasconi $\cdot$ M. Aassi

F. Hoffmann-La Roche, Basel, Switzerland

J. Sibilia

CHU Hautepierre, Strasbourg, France 
$43.5 \% / 23.8 \% / 10.0 \%$ vs $66.9 \% / 47.2 \% / 26.8 \% / 8.5 \%$, respectively; $p>0.12$ for all individual comparisons, including ACR50 propensity score analyses). The decrease in DAS28 was also similar between treatment groups (mean \pm standard deviation: $-3.41 \pm 1.49$ for tocilizumab monotherapy vs -3.43 \pm 1.43 for combination therapy; $p>0.33$ all analyses, including propensity score analyses). Tocilizumab had a comparable safety profile, and was similarly effective, when used as monotherapy or in combination with DMARDs in a broad population of patients with rheumatoid arthritis.

Keywords Disease activity · DMARDs (biologic) · DMARDs (synthetic) · Monotherapy • Open-label · Rheumatoid arthritis · Tocilizumab · Tumor necrosis factor- $\alpha$ inhibitor

\section{Introduction}

The current recommendation for patients with rheumatoid arthritis (RA) not responding adequately to methotrexate (MTX) or other disease-modifying antirheumatic drugs (DMARDs) is to coadminister a biologic [1]. However, approximately one-third of RA patients treated with biologics receive them as monotherapy (without DMARDs) [2]. Patients may discontinue DMARDs because of toxicity [3], contraindications, or personal choice [4].

The safety and efficacy of tocilizumab have been demonstrated in RA patients who have inadequate responses to MTX/ DMARDs (MTX/DMARD-IR) or tumor necrosis factor- $\alpha$ inhibitor (TNFi) agents (TNFi-IR) [5]. Tocilizumab monotherapy has been demonstrated to be more effective than MTX [6, 7] or DMARD [8] monotherapy. Further, tocilizumab combined with MTX has not been shown to be superior to tocilizumab monotherapy in MTX-IR patients [9]. In ACT-SURE, a previously reported nonrandomized, open-label study, tocilizumab was administered under conditions more typical of clinical practice [10]; investigators could administer tocilizumab as monotherapy or combined with DMARDs at their discretion if the patient was able to tolerate DMARDs. Here we describe and compare these two treatment approaches.

\section{Methods}

Study design

In this 24-week, phase $3 b$, single-arm, nonrandomized, openlabel safety and effectiveness study conducted July 2008 through March 2010, patients were enrolled from 264 centers in 25 countries. Patients were $\geq 18$ years of age, had moderate to severe active RA for $\geq 6$ months, and had inadequate clinical responses to $\geq 1$ DMARD and/or TNFi therapy. Tocilizumab $8 \mathrm{mg} / \mathrm{kg}$ was administered intravenously every 4 weeks. Patients receiving a TNFi agent before baseline (as monotherapy or combined with nonbiologic DMARDs) replaced it with tocilizumab; patients receiving DMARDs alone added tocilizumab to their regimens; and patients receiving a TNFi agent alone discontinued it and initiated tocilizumab monotherapy. DMARDs were maintained at stable doses (choice and dose at investigator discretion); if DMARDs were poorly tolerated (as determined by the investigator), tocilizumab was administered as monotherapy without a washout period. The DMARD dosage was modified (reduced) only for safety reasons. Oral corticosteroids $(\leq 10 \mathrm{mg} /$ day prednisone or equivalent) and nonsteroidal anti-inflammatory drugs (NSAIDs) had to be used at stable doses for at least 25 of 28 days before treatment (day 1). Oral corticosteroids were to be kept constant throughout the study unless tapering was required for safety reasons. If MTX-related adverse events (AEs) or laboratory abnormalities developed, dose reduction or change in route was considered for the patient before study withdrawal. Ethical and regulatory approval and patients' written informed consent were obtained in accordance with the Declaration of Helsinki; Good Clinical Practice was followed.

\section{Assessments}

Safety endpoints included AEs, serious AEs (SAEs), serious infections, neutrophil counts, and liver transaminase levels. Effectiveness endpoints included ACR20/50/70/90 responses, European League Against Rheumatism (EULAR) responses, DAS28 using erythrocyte sedimentation rate, and simplified and clinical disease activity index (SDAI and CDAI, respectively). Health Assessment Questionnaire-Disability Index (HAQ-DI) data were also collected. For individual components, missing data were imputed using last-observationcarried-forward until withdrawal for joint counts only; for ACR and EULAR responses, missing data were considered no response. Safety and effectiveness were assessed monthly.

\section{Statistical analyses}

In this exploratory analysis, which addresses prespecified protocol-defined study objectives, patients were grouped according to initial treatment: tocilizumab monotherapy (monotherapy group) or tocilizumab plus $\geq 1$ DMARD (combination group). Descriptive statistics were used for incidences of AEs and SAEs, and two-sided Clopper-Pearson $95 \%$ confidence intervals were calculated unless specified otherwise. To test the "tocilizumab monotherapy=combination" hypothesis for effectiveness in this nonrandomized setting, logistic or analysis of covariance (ANCOVA) models adjusted for previous treatment 
(DMARD-IR/TNFi-IR [TNFi previous use: $>2$ months before baseline vs TNFi recent use: $\leq 2$ months before baseline], known to have different efficacy outcomes [10]) were used with baseline DAS28, CDAI, or SDAI, as applicable, as relevant confounders. For two key endpoints, ACR50 response and DAS28 change, supportive post hoc analyses used propensity scores [11] computed using a logistic regression model (Electronic supplementary material (ESM) Table S1). Five matched groups were created based on quintiles of the score. Overall DAS28 difference and Cochran-Mantel-Haenszel statistics for ACR50 response were computed; propensity score was included as a covariate in multivariate models.

\section{Results}

Patients

The safety and intent-to-treat (ITT) populations included 1,681 patients (239 [14\%], monotherapy; 1,442 [86\%], combination therapy; ESM Fig. S1). Overall, patients had established RA (mean duration, 9.6 years) with high disease activity (mean DAS28, 6.0) and were highly treatment experienced (mean number of previous nonbiologic DMARDs [not including current treatment], 1.3; $42 \%$ had used TNFi agents [mean, 1.4]; Table 1). Disease duration and many baseline disease activity measures were higher in the monotherapy group, consistent with the fact that the majority $(72 \%)$ of monotherapy patients were TNFi-IR. In the combination group, MTX was the most common DMARD (79\%); $3 \%$ of monotherapy patients started a DMARD (all MTX) during the study.

\section{Safety}

The frequencies of AEs (82.4 vs $76.6 \%$ of patients in the monotherapy and combination groups) and AEs leading to withdrawal (5.4 vs $5.1 \%$ ) were similar between treatment groups (Table 2). The incidences of SAEs (19.4 vs 20.2/100 patient-years in monotherapy and combination therapy groups) and serious infections (4.6 vs 5.2/100 patient-years), which were the most common SAE, were also similar. Grade 3/4 neutropenia and transaminase elevations occurred less frequently with monotherapy than with combination therapy (treatment modifications after laboratory events were made according to the tocilizumab label). Three of four reported deaths occurred in the combination therapy group (Table 2 [10]).

\section{Effectiveness}

Eighty-seven percent of the ITT population completed the study (complete data for DAS28 and ACR core set (ESM Table S2) were available for 87 and $83 \%$ of patients, respectively). Percentages of patients achieving ACR20/50/ $70 / 90$ responses at week 24 were $66.9,46.6,26.4$, and $8.7 \%$, respectively, and were similar between treatment groups (maximum difference, $3.7 \% ; p>0.12$, all comparisons, including an analysis of ACR50 using propensity scores; Fig. 1a, ESM Table S1). ACR20/50/70 responses occurred as early as week 4 and improved through week 24 (ESM Fig. S2).

At week 24, no statistically significant difference was found between treatment groups in EULAR good/moderate responses, DAS28<2.6 (remission), or DAS28 low disease activity (LDA; Fig. 1b). EULAR good/moderate responses and DAS28<2.6 were achieved as early as week 4 , and percentages of patients achieving EULAR good/moderate responses were maintained through week 24 (ESM Figs. S3A, S3B). Percentages with DAS28<2.6 increased through weeks 20 and 24 in the monotherapy and combination groups, respectively (ESM Fig. S3B); overall, DAS28<2.6 was achieved in $56.8 \%$ of patients. At week 24 , numerically higher proportions of combination therapy patients than monotherapy patients achieved CDAI and SDAI LDA; CDAI and SDAI remission rates were similar (Fig. 1b), as were proportions of patients achieving ACR/EULAR Boolean remission (11.3\% monotherapy vs $11.9 \%$ combination; $p=$ 0.54 ). Decreases from baseline in DAS28 (mean \pm standard deviation, $-3.41 \pm 1.49$ monotherapy vs $-3.43 \pm 1.43$ combination), CDAI, and SDAI were also similar between treatment groups, with nonsignificant differences in univariate (Wilcoxon rank-sum test; Fig. 1c) or multivariate (ANCOVA) analyses, including analysis of DAS28 change using propensity scores ( $p>0.33$ for all analyses; ESM Table S1). Differences in effectiveness between treatment groups were small and nonsignificant in the subgroups of patients with and without previous TNFi therapy (Fig. 1c and d, ESM Table S3).

Decreases in joint counts from baseline to week 24 were similar between treatment groups (Fig. 1d). At week 24, decreases in HAQ-DI were comparable between groups (difference, $0.03 ; p=0.48$ ), but a higher percentage of combination therapy patients $(73.4 \%)$ than monotherapy patients $(68.4 \%)$ achieved clinically meaningful improvement in HAQ-DI ( $p=$ 0.030; ESM Fig. S4). Effectiveness outcomes in patients in the combination therapy group who received methotrexate (ESM Table S4) and in the monotherapy group were similar.

In a subset of study centers, patients who experienced at least moderate EULAR response after 24 weeks of treatment were eligible to enter a long-term extension study. Mean DAS28, DAS28 $<2.6$ responders, ACR20/50/70/90 responders, and percentages of patients who achieved clinically meaningful improvement in HAQ-DI were similar between treatment groups after 6 months of the extension study (ESM Table S5).

No evidence for an interaction between baseline DAS28 and treatment type was found using models for DAS28 and ACR20, 
Table 1 Baseline demographics and characteristics

\begin{tabular}{|c|c|c|c|c|}
\hline & $\begin{array}{l}\text { Tocilizumab monotherapy } \\
n=239\end{array}$ & $\begin{array}{l}\text { Tocilizumab+DMARD (s) } \\
n=1,442\end{array}$ & All patients $N=1,681$ & $p^{\mathrm{a}}$ \\
\hline Female, $\%$ & 82 & 81 & 81 & $0.79(\mathrm{~F})$ \\
\hline Age, years & $55.2(12.3)$ & $53.2(12.3)$ & $53.5(12.3)$ & $0.034(\mathrm{~W})$ \\
\hline Duration of RA, years & $11.0(9.7)$ & $9.4(8.6)$ & $9.6(8.8)$ & $0.0050(\mathrm{~W})$ \\
\hline DAS28 & $6.2(1.3)$ & $5.9(1.2)$ & $6.0(1.2)$ & $0.0010(\mathrm{~W})$ \\
\hline SJC & $14.0(10.7)$ & $12.6(8.9)$ & $12.8(9.2)$ & $0.22(\mathrm{~W})$ \\
\hline TJC & $25.5(15.9)$ & $22.4(14.8)$ & $22.8(15.0)$ & $0.0030(\mathrm{~W})$ \\
\hline Patient global VAS & $66.6(20.7)$ & $61.8(21.2)$ & $62.5(21.2)$ & $0.0015(\mathrm{~W})$ \\
\hline Physician global VAS & $62.4(18.4)$ & $58.2(17.7)$ & $58.8(17.9)$ & $0.0008(\mathrm{~W})$ \\
\hline Patient pain VAS & $61.3(22.5)$ & $56.9(22.6)$ & $57.5(22.6)$ & $0.0047(\mathrm{~W})$ \\
\hline $\mathrm{CRP}, \mathrm{mg} / \mathrm{dL}$ & $2.5(2.8)$ & $1.8(2.8)$ & $1.9(2.8)$ & $<0.0001(\mathrm{~W})$ \\
\hline $\mathrm{ESR}, \mathrm{mm} / \mathrm{h}$ & $44.6(28.5)$ & $38.3(26.5)$ & $39.2(26.8)$ & $0.0010(\mathrm{~W})$ \\
\hline HAQ-DI & $1.7(0.6)$ & $1.5(0.6)$ & $1.5(0.6)$ & $<0.0001(\mathrm{~W})$ \\
\hline CDAI & $36.5(15.0)$ & $34.0(13.4)$ & $34.3(13.6)$ & $0.019(\mathrm{~W})$ \\
\hline SDAI & $38.9(16.1)$ & $35.8(14.2)$ & $36.3(14.5)$ & $0.0047(\mathrm{~W})$ \\
\hline DMARD-IR, $n(\%)$ & $66(28)$ & $910(63)$ & $976(58)$ & $<0.0001(\mathrm{C})$ \\
\hline TNFi previous use, ${ }^{\mathrm{b}} n(\%)$ & $62(26)$ & $236(16)$ & $298(18)$ & \\
\hline TNFi recent use, ${ }^{\mathrm{c}} n(\%)$ & $111(46)$ & $296(21)$ & $407(24)$ & \\
\hline \multicolumn{5}{|l|}{ Baseline DMARD use, \% } \\
\hline MTX & 0 & 79 & 67 & \\
\hline Hydroxychloroquine & 0 & 16 & 14 & \\
\hline Sulfasalazine & 0 & 13 & 11 & \\
\hline Leflunomide & 0 & 13 & 11 & \\
\hline Baseline oral corticosteroid use, $n(\%)$ & $124(51.9)$ & $733(50.8)$ & $857(51.0)$ & \\
\hline Baseline oral corticosteroid dose, $\mathrm{mg} / \mathrm{d}^{\mathrm{d}}$ & $7.8(3.6)$ & $7.1(3.5)$ & $7.2(3.5)$ & \\
\hline \multicolumn{5}{|c|}{ Baseline DMARD dose } \\
\hline MTX, mg/week & 0 & $17.5(7.3)$ & $17.5(7.3)$ & \\
\hline Hydroxychloroquine, mg/day & 0 & $331.8(151.0)$ & $331.8(151.0)$ & \\
\hline Sulfasalazine, g/day & 0 & $1.9(0.6)$ & $1.9(0.6)$ & \\
\hline Leflunomide, mg/day & 0 & $18.4(4.6)$ & $18.4(4.6)$ & \\
\hline
\end{tabular}

Data are presented as mean $(S D)$ unless stated otherwise

VAS Visual Analogue Scale

${ }^{a}$ Between-group comparisons: $F$ Fisher exact test; $W$ Wilcoxon rank sum test; $C$ chi-square test for no association

${ }^{\mathrm{b}}$ Patients who did not use TNFi for $>2$ months before baseline

${ }^{\mathrm{c}}$ Patients who used TNFi for $\leq 2$ months before baseline

${ }^{\mathrm{d}}$ Dose in prednisone equivalents, considering only patients receiving corticosteroids

ACR50, and EULAR responses at week 24 with additional adjustment for previous treatment. This indicates that baseline disease activity is not a valuable response predictor for guiding a choice between monotherapy and combination therapy.

\section{Discussion}

In contrast to most phase 3 studies, ACT-SURE evaluated a population treated closer to actual clinical practice. Patients were from tertiary academic centers, nonacademic centers, and private practices with virtually no restrictions on previous DMARD and TNFi types, dosages, and combinations. Patients could be treated with a variety of concomitant medications including add-on DMARDs, NSAIDs, and corticosteroids. Our focus was on the comparison between TCZ used as monotherapy or in combination with nonbiologic DMARDs.

Relevant limitations of this study were the open-label design, which potentially introduced bias because clinical assessments were not performed in a blinded manner, the absence of randomization, the focus on short-term safety and 
clinical outcomes, and the discontinuation of DMARDs at study entry if poorly tolerated, at the discretion of the rheumatologist. Monotherapy patients had more severe disease at entry, reflecting the fact that this population consisted primarily of TNFi-IR patients. Reports indicate that approximately one-third of RA patients using biologics receive them as monotherapy [2]. This observation is in line with the rate of tocilizumab monotherapy in TNF-IR patients (25\%); however, only $7 \%$ of DMARD-IR patients received tocilizumab monotherapy as their first biologic. Additional factors such as lack of efficacy and poor adherence may also contribute to DMARD discontinuation over time during biologic treatment [12].

Slightly higher incidences of transaminase elevations and neutropenia with monotherapy were observed but were not associated with severe clinical consequences. Overall the safety profiles in patients receiving tocilizumab monotherapy and combination therapy overlapped, consistent with results of a recent double-blind study [9]. A larger study would be needed for an appropriate comparison of rates of uncommon events such as serious infections.

This exploratory analysis did not indicate a sizable difference in clinical effectiveness between tocilizumab monotherapy and combination therapy. To account for potential confounders, including previous treatments, multivariate analysis and propensity scores were used. Multivariate analysis confirmed previous reports from ACT-SURE suggesting that TNFi-IR patients represent a difficult-to-treat population in whom tocilizumab is slightly less effective than it is in TNF-naive patients [10]. However, in both TNFi-IR and DMARD-IR subpopulations, the effectiveness of monotherapy was comparable to that of combination therapy at week 24 . Retrospectively, this study would have had at least $80 \%$ power to detect a difference of 0.3 in DAS28 change and a 9.5\% ACR50 response difference. Thus, differences in effectiveness generally considered clinically relevant would likely have been identified.

These data are consistent with findings in other tocilizumab monotherapy trials. In MTX-naive or 6-month MTX-free patients and in MTX-IR patients, tocilizumab monotherapy resulted in higher ACR responses than MTX monotherapy [6, 8]. The efficacy of tocilizumab monotherapy was also demonstrated in a double-blind, head-to-head trial against adalimumab monotherapy in patients for whom MTX was considered inappropriate (because of lack of efficacy or intolerance) [13]. In most clinical trials, efficacy did not differ

Table 2 Safety outcomes

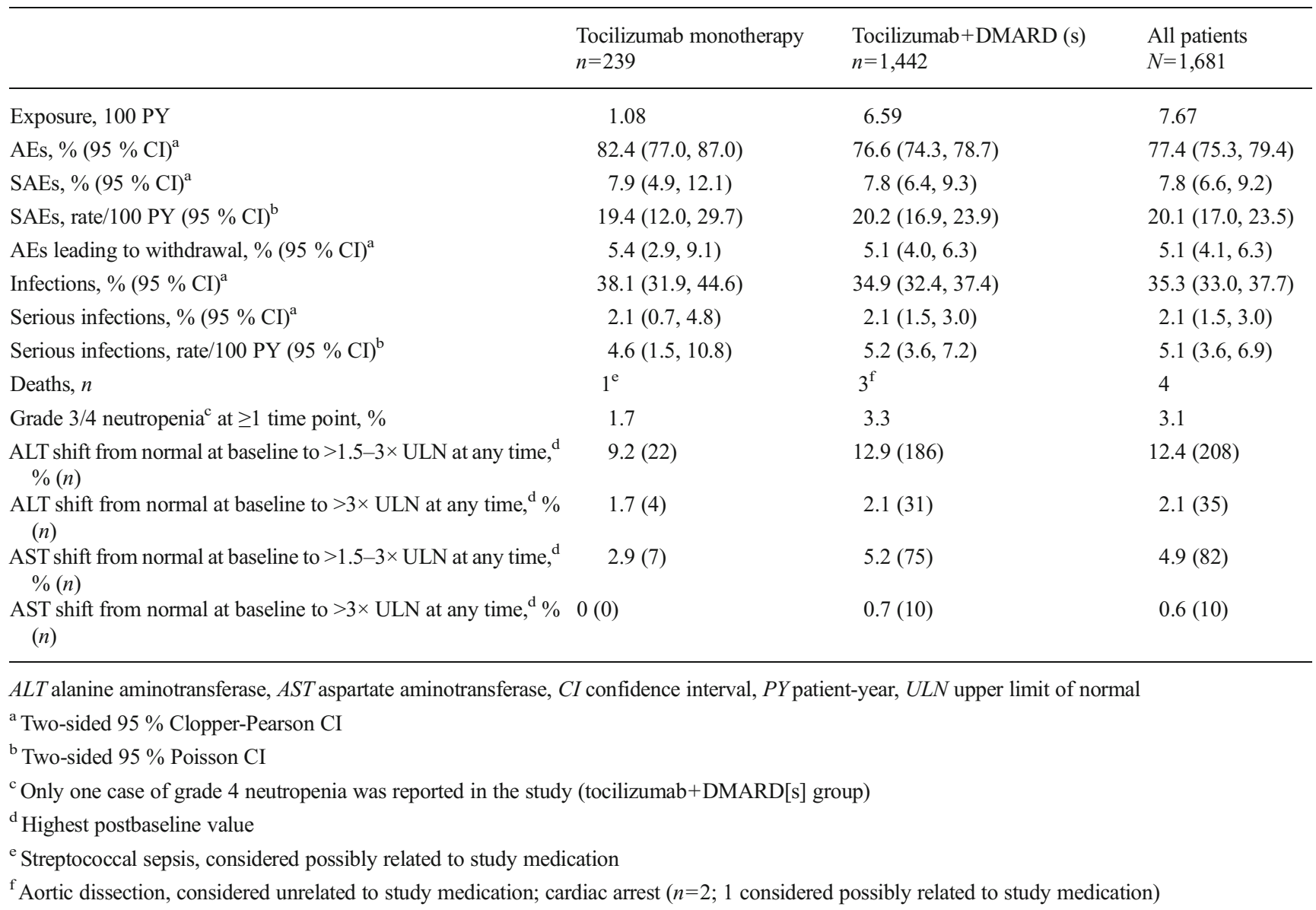




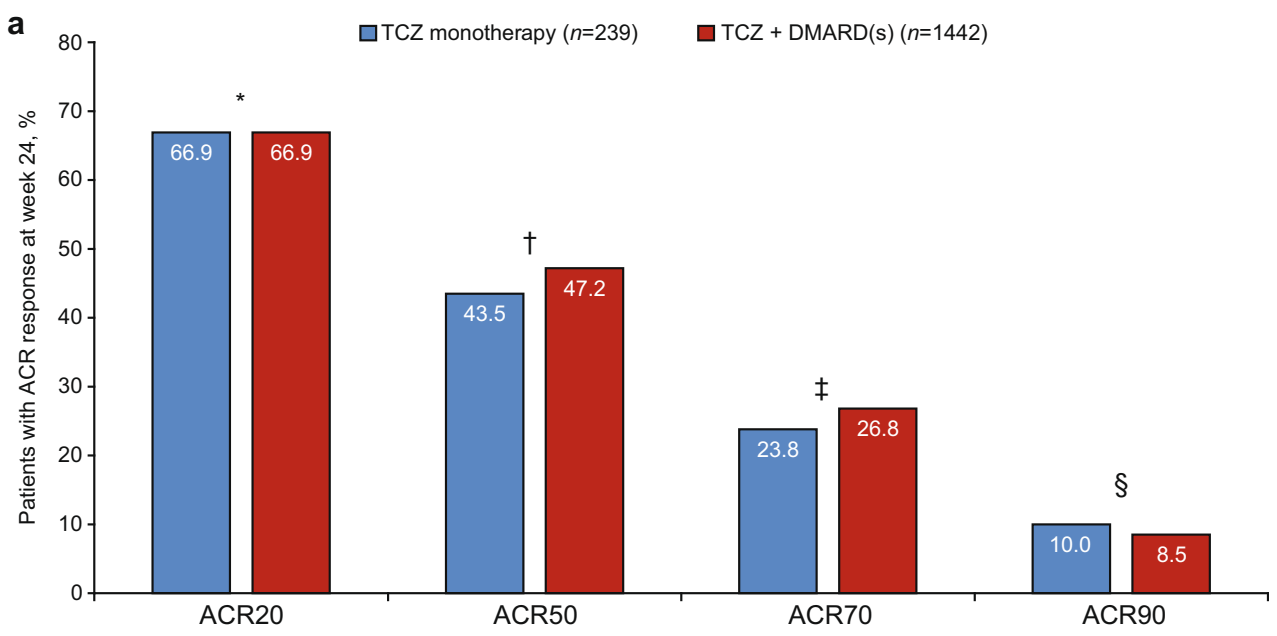

${ }^{*} p=0.51 ;{ }^{\dagger} p=0.95 ;{ }^{\dagger} p=0.66 ; \S_{p}=0.12$

b

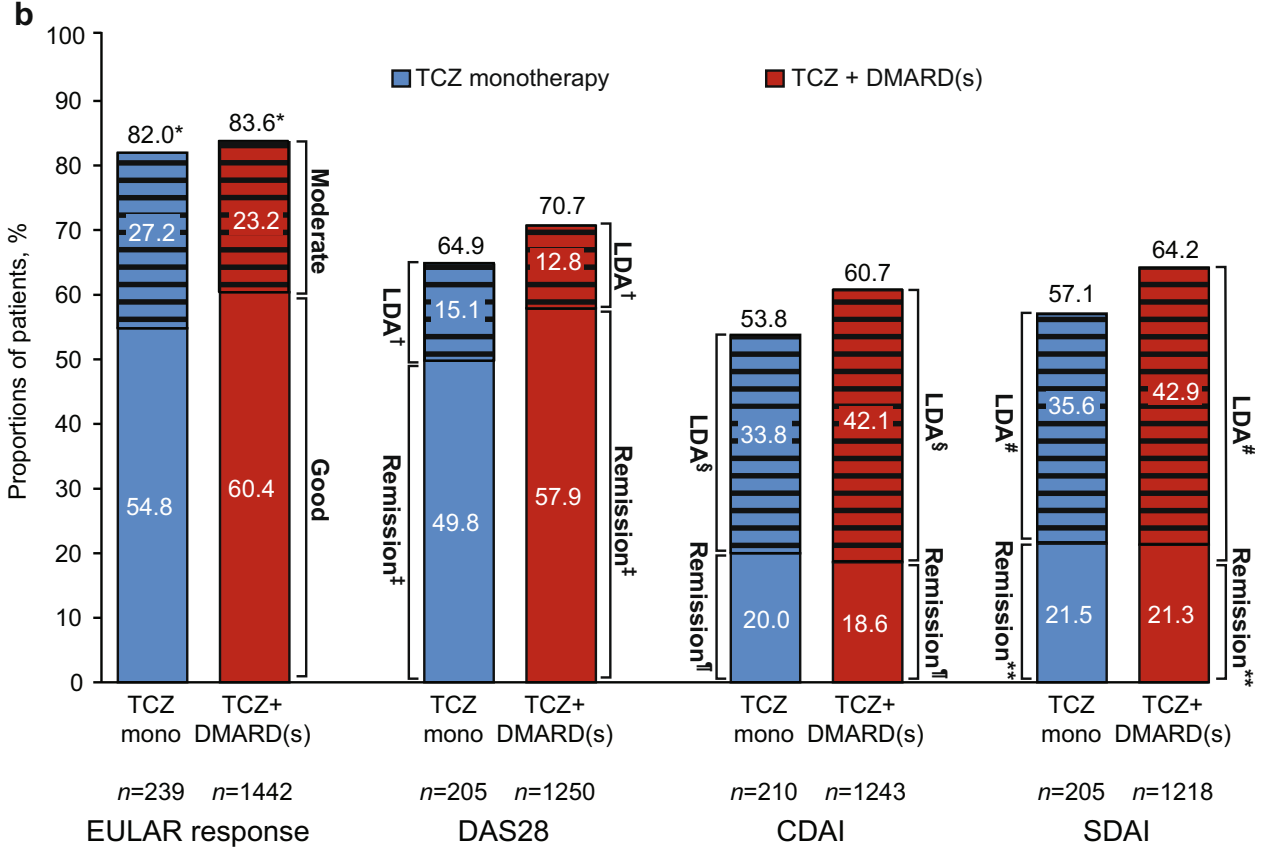

${ }^{*} p=0.95$ (good or moderate EULAR response); ${ }^{\dagger} p=0.59$ (includes remission);

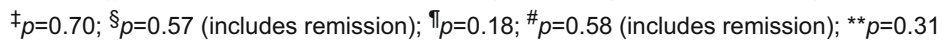

Fig. 1 Effectiveness at week 24. a ACR20/50/70/90 responses. b DAS28, CDAI, and SDAI responses. c Change from baseline in DAS28, CDAI, and SDAI. d Change from baseline in swollen joint count $(S J C)$ and tender joint count $(T J C)$. a $p$ values were calculated by logistic regression analysis adjusted for previous treatment (DMARD-IR/ TNFi-IR [previous TNFi use/recent TNFi use]) and baseline DAS28. Nonresponder imputation was performed for patients who withdrew or for whom responses were missing. $\mathbf{b}$ Hatched lines represent moderate EULAR response or low disease activity. EULAR good response: DAS2 $8 \leq 3.2$ at week 24 and change of $>-1.2$. EULAR moderate response: DAS2 $\leq \leq 3.2$ at week 24 and change of $<-0.6$ to $\geq-1.2$ or $<-1.2$; DAS28 $>3.2$ to $\leq 5.1$ at week 24 and change of $<1.2$. DAS28: low disease activity (LDA), $\geq 2.6$ to 3.2 ; remission, $<2.6$. CDAI: LDA, $>2.8$ to $\leq 10$; remission, $\leq 2.8$. SDAI: LDA, $>3.3$ to $\leq 11$; remission, $\leq 3.3$. $p$ values calculated by logistic regression analysis adjusted for previous treatment (DMARD-IR/TNFi-IR [previous TNFi use/recent TNFi use]) and baseline DAS28, CDAI, or SDAI, as applicable. c TCZ monotherapy in DMARD-IR patients, $n=66$; TCZ monotherapy in TNFi-IR patients, $n=173$; TCZ+DMARDs in DMARD-IR patients, $n=910$; TCZ+ DMARDs in TNFi-IR patients, $n=532$. $p$ values were calculated by Wilcoxon rank-sum test and compare TCZ monotherapy and TCZ combination therapy (disregarding the DMARD-IR-TNFi-IR split). d TCZ monotherapy in DMARD-IR patients, $n=66$; TCZ monotherapy in TNFi-IR patients, $n=173$; TCZ+DMARDs in DMARD-IR patients, $n=910$; TCZ+DMARDs in TNFi-IR patients, $n=532 ; p$ values were calculated by Wilcoxon rank-sum test and compare TCZ monotherapy and TCZ combination therapy (disregarding the DMARD-IR-TNFi-IR split) 


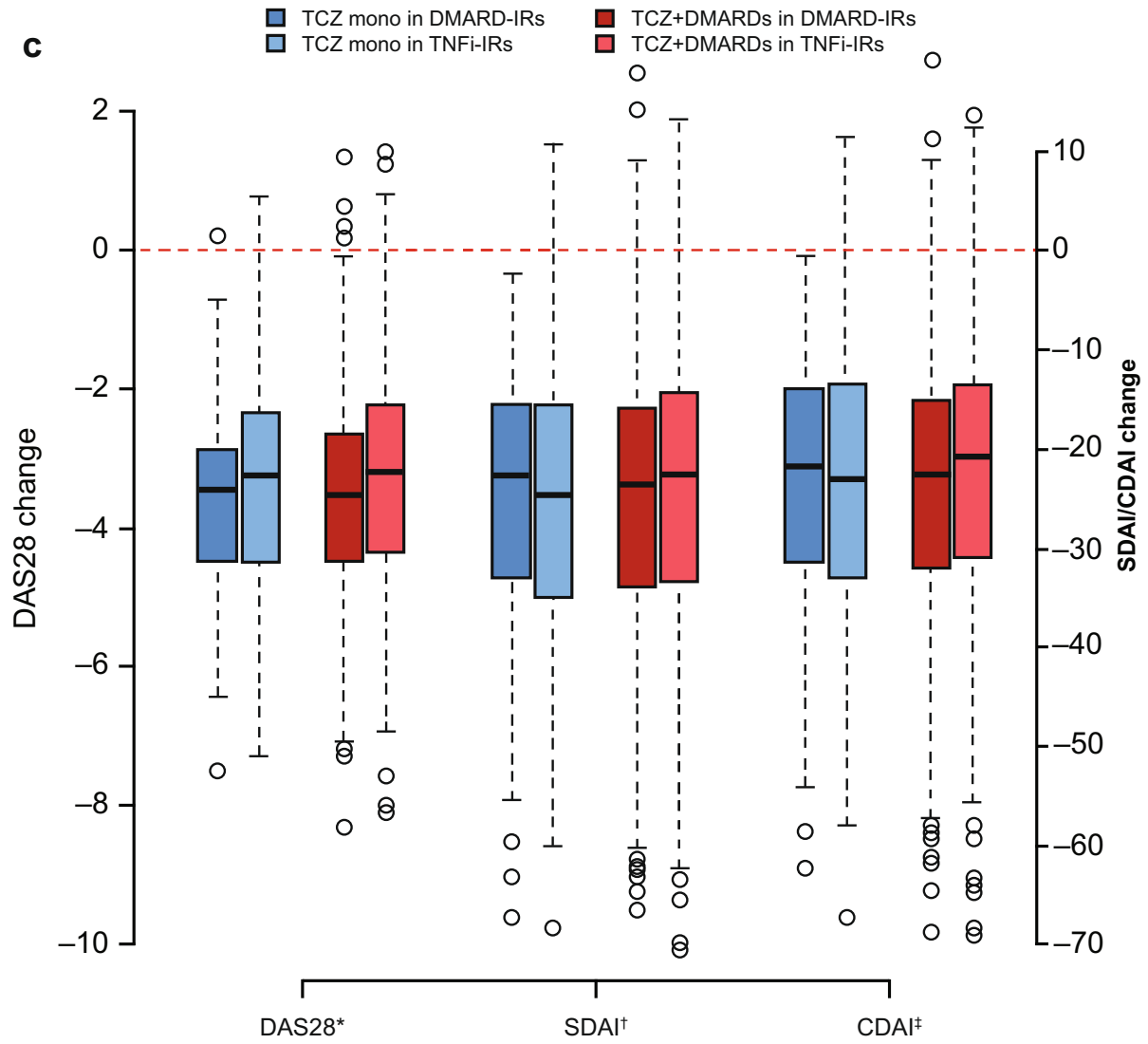

${ }^{*} p=0.9 ;{ }^{\dagger} p=0.45 ;{ }^{\ddagger} p=0.85$

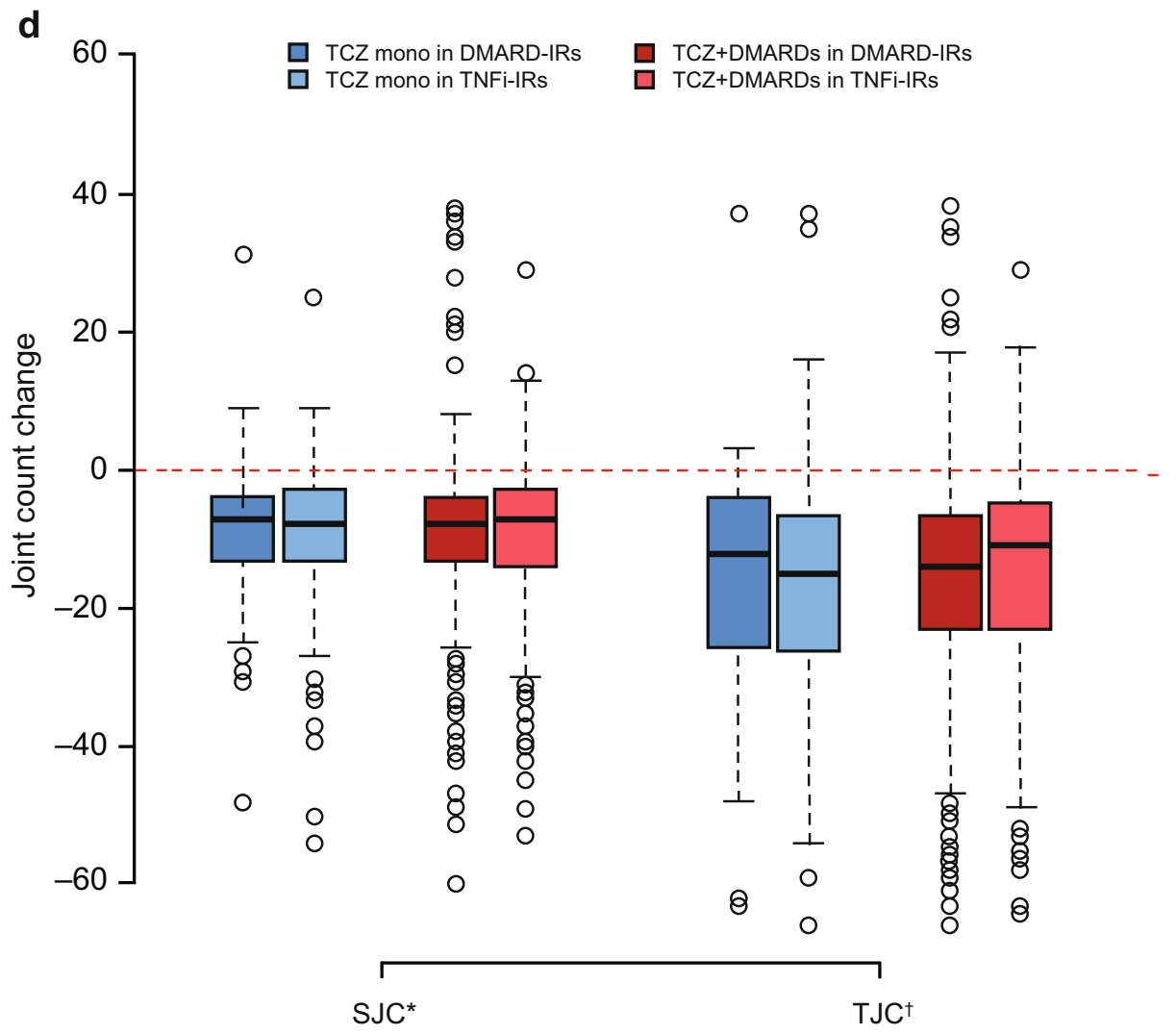

Fig. 1 (continued)

${ }^{*} p=0.99 ;{ }^{\dagger} p=0.27$ 
substantially between tocilizumab monotherapy and combination therapy at 6 months, whereas long-term comparative data are lacking. Further studies are needed to confirm the sustained response to tocilizumab monotherapy in RA patients who experienced inadequate response to previous treatments. In the 24-week analysis of a randomized study in 556 MTX-IR patients [9], no clinically relevant superiority was demonstrated with an MTX plus tocilizumab add-on strategy over switching to tocilizumab monotherapy. Small numerical differences in the primary and some secondary endpoints favoring combination therapy were not considered clinically meaningful. Similarly, in an open-label, randomized study in DMARD-IR or DMARD-intolerant patients, efficacy was comparable to that of tocilizumab monotherapy and combination therapy [14]. In two reports, combination therapy with MTX appeared more effective than tocilizumab monotherapy. However, one study [15] was a small dose-finding study in MTX-IR patients, and the other study [16] was a retrospective analysis of clinical practice in Japan. In the latter, results may be explained by differences in baseline characteristics, for which estimates were not adjusted; in particular, disease activity, a clear predictor of lower remission rates, was higher for monotherapy patients than for combination therapy patients [16].

Monotherapy with TNFi agents has also been studied. Results from ReACT, a study of adalimumab with a design similar to that of ACT-SURE, showed larger differences between TNFi monotherapy and combination therapy [17]. In addition, randomized controlled trials indicate that a TNFi agent plus MTX is more effective than either alone [18-20].

In summary, in this 6-month study, tocilizumab had a comparable safety profile and was similarly effective when used as monotherapy or as combination therapy with DMARDs in a broader population of patients. These data further support that tocilizumab monotherapy is a feasible treatment alternative for patients for whom combination therapy with MTX is not an option.

Acknowledgments Funding for manuscript preparation was provided by F. Hoffmann-La Roche. The authors thank Sara Duggan and Meryl Mandle, who provided writing services on behalf of F. Hoffmann-La Roche. This work was supported by F. Hoffmann-La Roche. Maher Aassi is an employee of and Corrado Bernasconi is a contractor to F. HoffmannLa Roche. They participated in the study design, in the collection, analysis, and interpretation of data, in drafting the manuscript, and in the decision to submit the paper for publication.

Conflict of interest Vivian P. Bykerk: consulting fees from Roche and Genentech as well as BMS, UCB, Amgen, Pfizer, Merck, Antares. Andrew J. K. Östör: consulting and expert testimony fees for expert opinion, honoraria for lectures; fees for the development of educational presentations and aids; and travel expenses to attend conferences from all or some of the following: Roche, Chugai, Schering-Plough/MSD, AbbVie, Wyeth/Pfizer, BMS, GSK, MerckSerono, and UCB. José Alvaro-Gracia: consulting fees from Roche, BMS, UCB, and Pfizer/ Wyeth; lecture/speakers bureau fees from Roche, BMS, UCB, Pfizer/
Wyeth, MSD/Schering-Plough, and AbbVie; travel expenses from Roche; and grants to his institution from Roche. Karel Pavelka: consulting fees and lecturer/speaker fees from Roche, AbbVie, BMS, Pfizer, Amgen, and MSD. José Andrés Román-Ivorra: advisory boards for BMS, Amgen, and Pfizer; research grants from MSD, UCB, and Roche; honoraria from Amgen, BMS, and Roche (for educational presentations); travel expenses from MSD and Pfizer. Winfried Graninger: board member, consulting, lecture/speaker and travel fees from Roche; grants and case compensation to institution from Roche. William Bensen: speaker fees from Roche. Michael T. Nurmohamed: consulting fees from Roche, Schering-Plough, MSD, BMS, UCB, Wyeth, Pfizer, and Sobi; speaker/ lecture fees from AbbVie, Roche, BMS, and Pfizer; travel expenses from Roche, Abbott, UCB, and MSD; grants to institution from Roche, AbbVie, UCB, and Pfizer. Andreas Krause: honoraria, lecture/speaker fees and travel expenses from AbbVie, Roche, Pfizer, MSD, BMS, UCB (self and institution). Corrado Bernasconi: consulting fees from Roche Global Medical Affairs. Maher Aassi: employee of Roche, Basel, Switzerland. Jean Sibilia: board membership and lecture/speaker fees from Roche, MSD, AbbVie, Pfizer, Actelion, BMS, Novartis, and Amgen.

Open Access This article is distributed under the terms of the Creative Commons Attribution License which permits any use, distribution, and reproduction in any medium, provided the original author(s) and the source are credited.

\section{References}

1. Smolen JS, Landewe R, Breedveld FC, Dougados M, Emery P, Gaujoux-Viala C, Gorter S, Knevel R, Nam J, Schoels M, Aletaha D, Buch M, Gossec L, Huizinga T, Bijlsma JW, Burmester G, Combe B, Cutolo M, Gabay C, Gomez-Reino J, Kouloumas M, Kvien TK, Martin-Mola E, McInnes I, Pavelka K, van Riel P, Scholte M, Scott DL, Sokka T, Valesini G, van Vollenhoven R, Winthrop KL, Wong J, Zink A, van der Heijde D (2010) EULAR recommendations for the management of rheumatoid arthritis with synthetic and biological disease-modifying antirheumatic drugs. Ann Rheum Dis 69:964-975

2. Soliman MM, Ashcroft DM, Watson KD, Lunt M, Symmons DP, Hyrich KL (2011) Impact of concomitant use of DMARDs on the persistence with anti-TNF therapies in patients with rheumatoid arthritis: results from the British Society for Rheumatology Biologics Register. Ann Rheum Dis 70:583-589

3. Salliot C, Van Der Heijde D (2009) Long term safety of methotrexate monotherapy in rheumatoid arthritis patients: a systematic literature research. Ann Rheum Dis 68:1100-1104

4. Kinder AJ, Hassell AB, Brand J, Brownfield A, Grove M, Shadforth MF (2005) The treatment of inflammatory arthritis with methotrexate in clinical practice: treatment duration and incidence of adverse drug reactions. Rheumatology (Oxford) 44:61-66

5. Navarro-Millan I, Singh JA, Curtis JR (2012) Systematic review of tocilizumab for rheumatoid arthritis: a new biologic agent targeting the interleukin-6 receptor. Clin Ther 34:788-802

6. Jones G, Sebba A, Gu J, Lowenstein MB, Calvo A, Gomez-Reino JJ, Siri DA, Tomsic M, Alecock E, Woodworth T, Genovese MC (2010) Comparison of tocilizumab monotherapy versus methotrexate monotherapy in patients with moderate to severe rheumatoid arthritis: the AMBITION study. Ann Rheum Dis 69:88-96

7. Nishimoto N, Hashimoto J, Miyasaka N, Yamamoto K, Kawai S, Takeuchi T, Murata N, van der Heijde D, Kishimoto T (2007) Study of active controlled monotherapy used for rheumatoid arthritis, an IL6 inhibitor (SAMURAI): evidence of clinical and radiographic 
benefit from an $\mathrm{x}$ ray reader-blinded randomised controlled trial of tocilizumab. Ann Rheum Dis 66:1162-1167

8. Nishimoto N, Miyasaka N, Yamamoto K, Kawai S, Takeuchi T, Azuma J, Kishimoto T (2009) Study of active controlled tocilizumab monotherapy for rheumatoid arthritis patients with an inadequate response to methotrexate (SATORI): significant reduction in disease activity and serum vascular endothelial growth factor by IL-6 receptor inhibition therapy. Mod Rheumatol 19:12-19

9. Dougados M, Kissel K, Sheeran T, Tak PP, Conaghan PG, Mola EM, Schett G, Amital H, Navarro-Sarabia F, Hou A, Bernasconi C, Huizinga TW (2013) Adding tocilizumab or switching to tocilizumab monotherapy in methotrexate inadequate responders: 24-week symptomatic and structural results of a 2 year randomized controlled strategy trial in rheumatoid arthritis (ACT-RAY). Ann Rheum Dis 72:43-50

10. Bykerk V, Ostör AJ, Alvaro-Gracia J, Pavelka K, Ivorra JA, Graninger W, Bensen W, Nurmohamed MT, Krause A, Bernasconi C, Stancati A, Sibilia J (2012) Tocilizumab in patients with active rheumatoid arthritis and inadequate responses to DMARDs and/or TNF inhibitors: a large, open-label study close to clinical practice. Ann Rheum Dis 71:1950-1954

11. D'Agostino RB Jr (1998) Propensity score methods for bias reduction in the comparison of a treatment to a non-randomized control group. Stat Med 17:2265-2281

12. de Thurah A, Norgaard M, Johansen MB, Stengaard-Pedersen K (2010) Methotrexate compliance among patients with rheumatoid arthritis: the influence of disease activity, disease duration, and comorbidity in a 10-year longitudinal study. Scand J Rheumatol 39: 197-205

13. Gabay C, Emery P, van Vollenhoven R, Dikranian A, Alten R, Pavelka K, Klearman M, Musselman D, Agarwal S, Green J, Kavanaugh A, ADACTA Study Investigators (2013) Tocilizumab monotherapy versus adalimumab monotherapy for treatment of rheumatoid arthritis (ADACTA): a randomised, double-blind, controlled phase 4 trial. Lancet 381:1541-1550

14. Weinblatt ME, Kremer J, Cush J, Rigby W, Teng LL, Devenport J, Singh N, Lepley D, Genovese MC (2013) Tocilizumab as monotherapy or in combination with nonbiologic DMARDs: 24-week results of an open-label, clinical practice study (ACT-STAR). Arthritis Care Res 65:362-371

15. Maini RN, Taylor PC, Szechinski J, Pavelka K, Bröll J, Balint G, Emery P, Raemen F, Petersen J, Smolen J, Thomson D, Kishimoto T, CHARISMA Study Group (2006) Double-blind randomized controlled clinical trial of the interleukin-6 receptor antagonist, tocilizumab, in European patients with rheumatoid arthritis who had an incomplete response to methotrexate. Arthritis Rheum 54:2817-2829

16. Yamanaka H, Tanaka Y, Inoue E, Hoshi D, Momohara S, Hanami K, Yunoue N, Saito K, Amano K, Kameda H, Takeuchi T (2011) Efficacy and tolerability of tocilizumab in rheumatoid arthritis patients seen in daily clinical practice in Japan: results from a retrospective study (REACTION study). Mod Rheumatol 21:122-133

17. Burmester GR, Mariette X, Montecucco C, Monteagudo-Sáez I, Malaise M, Tzioufas AG, Bijlsma JW, Unnebrink K, Kary S, Kupper H (2007) Adalimumab alone and in combination with disease-modifying antirheumatic drugs for the treatment of rheumatoid arthritis in clinical practice: the Research in Active Rheumatoid Arthritis (ReAct) trial. Ann Rheum Dis 66:732-739

18. Breedveld FC, Weisman MH, Kavanaugh AF, Cohen SB, Pavelka K, van Vollenhoven R, Sharp J, Perez JL, Spencer-Green GT (2006) The PREMIER study: a multicenter, randomized, double-blind clinical trial of combination therapy with adalimumab plus methotrexate versus methotrexate alone or adalimumab alone in patients with early, aggressive rheumatoid arthritis who had not had previous methotrexate treatment. Arthritis Rheum 54:26-37

19. Van Der Heijde D, Klareskog L, Landewé R, Bruyn GA, Cantagrel A, Durez P, Herrero-Beaumont G, Molad Y, Codreanu C, Valentini G, Zahora R, Pedersen R, MacPeek D, Wajdula J, Fatenejad S (2007) Disease remission and sustained halting of radiographic progression with combination etanercept and methotrexate in patients with rheumatoid arthritis. Arthritis Rheum 56:3928-3939

20. Kremer J, Ritchlin C, Mendelsohn A, Baker D, Kim L, Xu Z, Han J, Taylor P (2010) Golimumab, a new human anti-tumor necrosis factor alpha antibody, administered intravenously in patients with active rheumatoid arthritis: forty-eight-week efficacy and safety results of a phase III randomized, double-blind, placebo-controlled study. Arthritis Rheum 62:917-928 